\title{
DIATOMEAS (BACILLARIOPHYCEAE) DE ZONAS ANDINAS DEL NORTE DE CHILE: NUEVA LOCALIDAD GEOGRÁFICA PARA HALOROUNDIA SPECIOSA (HUSTEDT) DIAZ ET MAIDANA
}

\author{
DIATOM TAXA (BACILLARIOPHYCEAE) FROM ANDEAN AREAS \\ IN NORTHERN CHILE: NEW GEOGRAPHICAL LOCALITY FOR \\ HALOROUNDIA SPECIOSA (HUSTEDT) DIAZ ET MAIDANA
}

\author{
Patricio Rivera \& Fabiola Cruces \\ Departamento de Botánica, Facultad de Ciencias Naturales y Oceanográficas, \\ Universidad de Concepción, Casilla 160-C, Concepción, Chile. \\ privera@udec.cl
}

ABSTRACT

\begin{abstract}
This note reports the first finding of Haloroundia speciosa (Hustedt) Díaz et Maidana (Bacillariophyceae) in a sample

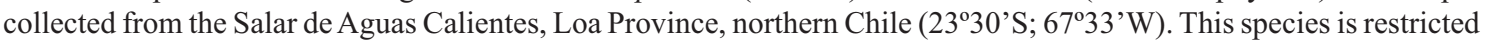
to the Andes Mountains. Comments about its morphological features and geographical distribution in north Andean areas are given.
\end{abstract}

Durante las últimas décadas el género Achnanthes Bory ha sido objeto de varias revisiones taxonómicas basadas en sus características morfológicas, y como consecuencia de las mismas, muchas de sus especies, distribuidas fundamentalmente en los cuerpos de aguas continentales, han sido transferidas a nuevos géneros (Lange-Bertalot \& Krammer 1989, Round et al. 1990, Bukhtiyarova \& Round 1996, Round \& Bukhtiyarova 1996).

En 1927, Hustedt describió a Achnanthes speciosa sobre la base de material recolectado en el Río Loa, Desierto de Atacama, Chile, y recientemente Díaz et Maidana (2006) crearon el género Haloroundia sobre la base de la morfología de esta especie. Las autoras consideraron que características relacionadas con los plastidios, estrías, rafe y estructura de las bandas del cíngulum de esta especie justificaban tal separación.

El estudio de material diatomológico proveniente del Salar de Aguas Calientes, Chile, depositado en la Colección Diatomológica de la Universidad de Concepción, Chile, llevado a cabo mediante técnicas de microscopía fotónica y electrónica de barrido, reveló la presencia de más de una decena de valvas de este taxón, el cual no había sido señalado anteriormente para este lugar. El Salar de Aguas Calientes (233'ㅇ' 67³3'W) está ubicado en la II Región de Antofagasta, Provincia del Loa, a 4.200 m s.n.m. Es un salar con una superficie de $134 \mathrm{~km}^{2}$ en una cuenca de 1.168 $\mathrm{km}^{2}$, que posee costras salinas duras compuestas por sulfato de calcio y cloruro de sodio (Vila 1986); presenta varias lagunas de extensión variable y los aportes superficiales más importantes son el Río Pili y la Quebrada de Chamaca (Risacher et al. 1999).

Las valvas de $H$. speciosa observadas en el material del Salar de Aguas Calientes son elípticolanceoladas con extremos producidos, redondeados, de 26-48 $\mu \mathrm{m}$ de largo y 10-16 $\mu \mathrm{m}$ de ancho (Fig.1 A-D). La estructura, tamaño, orientación y densidad de las estrías es similar en las valvas provistas y carentes de rafe. Estas son marginales, 13-15 en $10 \mu \mathrm{m}$, levemente radiales (Fig.1 AG) y con estructura alveolada: externamente presentan tres líneas de aréolas (Fig.1 B-C, EG) y hacia el interior un gran foramen (Fig. $1 \mathrm{~F}$ ). Debido al reducido tamaño de las estrías (1,25- 
4,5 $\mu \mathrm{m}$ de largo), el sternum y el raphe sternum es enorme y de contorno anchamente lanceolado (Fig.1 B-D). El rafe es filiforme, derecho (Fig. 1 A-B); externamente los extremos proximales son ensanchados, lanceolados y bastante distanciados entre sí (Fig. 1 B, E), internamente existe una notoria depresión de la pared silícea (Fig.1 F); los extremos distales se curvan en la misma dirección (Fig. 1 B). Estas características concuerdan con las indicadas por Hustedt (1927) en la descripción original de
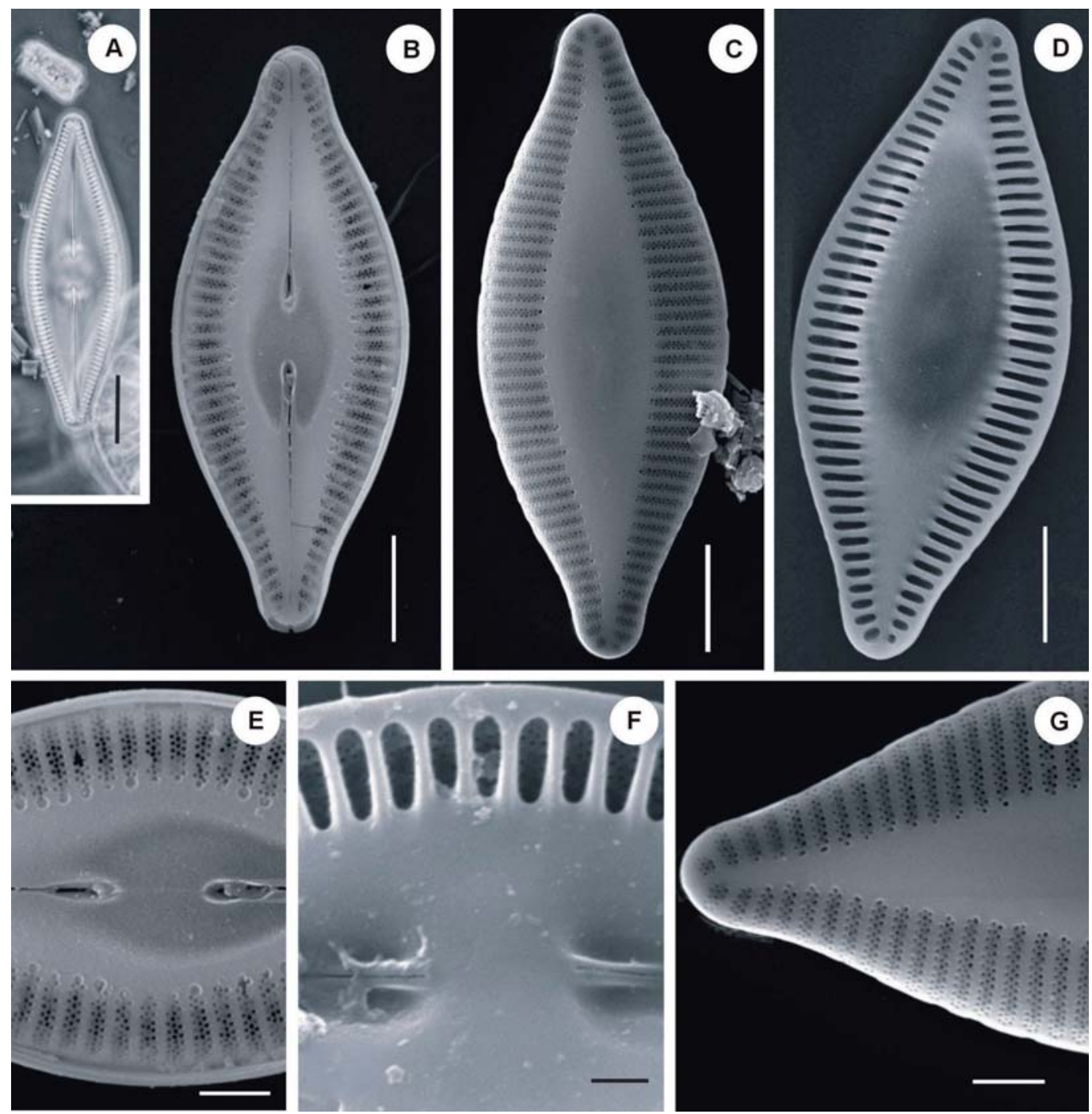

Figura 1. Haloroundia speciosa (Hustedt) Díaz et Maidana. Salar de Aguas Calientes, Chile, 20.VII.2002. Fig. A, LM; Figs. B-G SEM. A-D: valvas elíptico-lanceoladas con extremos diferenciados; sternum y raphe sternum amplios, lanceolados; estrías marginales levemente radiales. E: vista externa de los extremos proximales del rafe, ensanchados y distantes entre sí; tres líneas de aréolas en cada estría. F: vista interna de los extremos proximales del rafe con una depresión silícea; note los alvéolos marginales. G: vista externa de un extremo valvar; estrías con tres líneas de aréolas. Escala: $\mathrm{A}=10 \mu \mathrm{m}, \mathrm{B}-\mathrm{D}=5 \mu \mathrm{m}, \mathrm{E}, \mathrm{G}=2 \mu \mathrm{m}, \mathrm{F}=1 \mu \mathrm{m}$.

Figure 1. Haloroundia speciosa (Hustedt) Díaz et Maidana. Salar de Aguas Calientes, Chile, 20.VII.2002. Fig. A, LM; Figs. B-G, SEM. A-D: elliptic-lanceolate valves with protruded ends; broad lanceolate sternum and raphe sternum; slightly radial marginal striae. E: external view of enlarged and faraway proximal raphe endings; three lines of areolae on each stria. F: internal view of proximal raphe endings with a silica depression; note marginal alveoli. G: external view of a valve end; striae with three lines of areolae. Scale bar: $A=10 \mu \mathrm{m}, \mathrm{B}-\mathrm{D}=5 \mu \mathrm{m}, \mathrm{E}, \mathrm{G}=2 \mu \mathrm{m}, \mathrm{F}=1 \mu \mathrm{m}$. 
Achnanthes speciosa y por Díaz \& Maidana (2006) al describir el género Haloroundia.

Haloroundia speciosa parece ser una especie endémica de ambientes salinos de la Cordillera de Los Andes, y hasta el momento se le conoce una distribución en Chile entre los $18^{\circ} 50^{\prime} \mathrm{S}$ y los $25^{\circ} 80^{\prime} \mathrm{S}$ y desde los 1.030 a los 4.260 metros sobre el nivel del mar. Aparte de la localidad tipo - Río

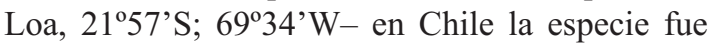
también señalada por Frenguelli (1929) para el

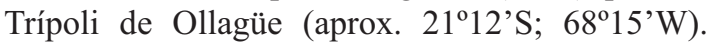
Rumrich et al. (2000) ilustran cuatro fotografías del taxón obtenidas con microscopía fotónica del material recolectado en "Grosser Norden, Río Loa, bei Paso del Toco, fóssil”, ubicación que corresponde exactamente a la localidad tipo de Hustedt. Los autores no indican si se trata de una revisión de ese material depositado en la Colección de Hustedt, Alemania, o si pudieron acceder a la misma localidad geográfica. Díaz \& Maidana (2006) lo encontraron en material de los salares

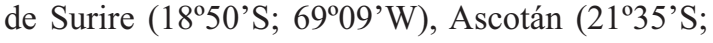
$\left.68^{\circ} 18^{\prime} \mathrm{W}\right)$, Atacama (2200'S; 69 $\left.35^{\circ} \mathrm{W}\right)$, Punta

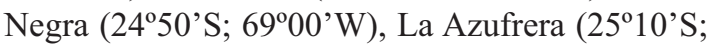
$\left.68^{\circ} 50^{\prime} \mathrm{W}\right)$ y La Isla $\left(25^{\circ} 80^{\prime} \mathrm{S} ; 6^{\circ} 60^{\prime} \mathrm{W}\right)$. También fue señalada por Maidana \& Seeligmann (2006) para la localidad argentina de Laguna Pasto Ventura, Provincia de Catamarca, a 3.700 m s.n.m.

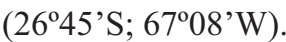

En el Salar de Aguas Calientes Haloroundia speciosa fue escasa en la muestra analizada, y estuvo acompañada de representantes de los géneros Mastogloia Thwaites, Denticula Kützing, Cymbella Agardh, Frankophila Lange-Bertalot, Navicula Bory y Surirella Turpin (trabajo en preparación).

Material estudiado: DiAT-CONC 3299, Salar de Aguas Calientes, $23^{\circ} 30^{\prime} \mathrm{S}$; $67^{\circ} 33^{\prime} \mathrm{W}$, s/c, 20-VII2002, 4220 m s.n.m.

\section{AGRADECIMIENTOS}

Agradecemos a la Dra. Irma Vila por el aporte de material diatomológico a la Colección de Diatomeas de la Universidad de Concepción, Chile, y a dos revisores anónimos por sus comentarios al manuscrito. Agradecemos también las facilidades otorgadas por el Departamento de Botánica y por el personal de Laboratorio de Microscopía Electrónica de la Universidad de Concepción, Chile.

\section{BIBLIOGRAFIA}

Bukhtiyarova, L. \& F.E. Round. 1996. Revision of the genus Achnanthes sensu lato section Marginulatae Bukht. Sect. nov. of Achnanthidium Kütz. Diatom Research 11(1): 1-30.

Díaz, C.A. \& N.I. Maidana. 2006. A new monoraphid diatom genus: Haloroundia Díaz \& Maidana. Nova Hedwigia, Beih. 130: 177-184.

Frenguelli, J. 1929. Diatomee fossili delle conche saline del deserto cileno-boliviano. Bolletino della Societa Geologica Italiana 47: 185-236.

Hustedt, F. 1927. Fossile Bacillariaceen aus dem LoaBecken in der Atacama-Wüste, Chile. Archiv für Hydrobiologie und Planktonkunde 18(2): 224-251.

Lange-Bertalot, H. \& K.D. Krammer. 1989. Achnanthes eine Monographie der Gattung mit Definition der Gattung Cocconeis. Bibliotheca Diatomologica Vol. 18, 385 S. J. Cramer, Berlin \& Sttutgart.

Maidana, N.I. \& C. Seeligmann. 2006. Diatomeas (Bacillariophyceae) de Ambientes Acuáticos de Altura de la Provincia de Catamarca, Argentina II. Boletín de la Sociedad Argentina de Botánica 41(1): 1-13.

Risacher, F., Alonso, H. \& C. Salazar. 1999. Geoquímica de aguas en cuencas cerradas: I, II y III Regiones, Chile. Ministerio de Obras Públicas-Dirección General de Aguas, Universidad Católica del Norte, Institut de Recherche pour le Développement, Convenio de Cooperación DGA-UCN-IRD. Volumen 3, pp. II145-II154.

Round, F.E., R.M. Crawford \& D.G. Mann. 1990. The Diatoms. Biology and morphology of the genera. Cambridge (Cambridge University Press). 747 pp.

Round, F.E. \& L. Bukhtiyarova. 1996. Four new genera based on Achnanthes (Achnanthidium) together with a re-definition of Achnanthidium. Diatom Research 11(2): 345-361.

Rumrich, U., H. Lange-Bertalot \& M. Rumrich. 2000. Diatomeen der Anden (Von Venezuela bis Patagonien/Tierra del Fuego und zwei weitere Beiträge). In: H. Lange-Bertalot (ed.). Iconographia Diatomologica, Vol 9, $673 \mathrm{~S}$.

VILA, T. 1986. Geología de los depósitos salinos del Norte de Chile. Antecedentes geoquímicos y pautas de prospección del Litio. En: J. Frutos, R. Oyarzún, \& M. Pincheira (eds.), Geología de Recursos Minerales de Chile. Tomo II, pp. 801819. Ed. Universidad de Concepción, Chile. 